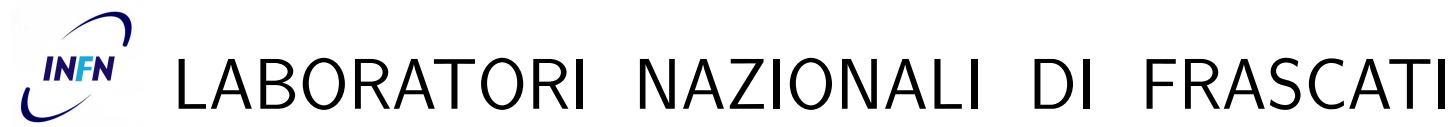 SIS-Pubblicazioni
}

LNF-08/29(P)

November 29, 2008

\section{THE CMS RPC GAS GAIN MONITORING SYSTEM: an Overview and Preliminary Results}

\author{
L. Benussi ${ }^{1}$, S. Bianco ${ }^{1}$, S. Colafranceschi ${ }^{1}, 2,3$, D. Colonna ${ }^{1}$, L. Daniello ${ }^{1}$, F. L. Fabbri ${ }^{1}$, M. Giardoni ${ }^{1}$ \\ B. Ortenzi ${ }^{1}$, A. Paolozzi, ${ }^{1}, 2$ L. Passamonti ${ }^{1}$, D. Pierluigi ${ }^{1}$ \\ B. Ponzio ${ }^{1}$, C. Pucci ${ }^{1}$, A. Russo ${ }^{1}$, G. Roselli ${ }^{5}$, A. Colaleo ${ }^{4}$, F. Loddo $^{4}$, M. Maggi ${ }^{4}$ \\ A. Ranieri ${ }^{4}$, M. Abbrescia ${ }^{4}, 5$, G. Iaselli ${ }^{4}, 5$, B. Marangelli ${ }^{4}, 5$, S. Natali $^{4}, 5$

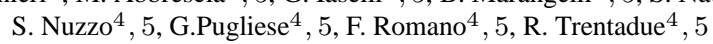 \\ S. Tupputi ${ }^{4}, 5$, R. Guida ${ }^{3}$, G. Polese ${ }^{3}, 6$, N. Cavallo ${ }^{7}$ A. Cimmino ${ }^{7}, 8$

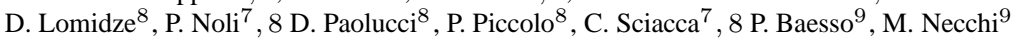 \\ D. Pagano ${ }^{9}$, S. P. Ratti ${ }^{9}$, P. Vitulo ${ }^{9}$, C. Viviani ${ }^{9}$ \\ 1) INFN Laboratori Nazionali di Frascati, Via E. Fermi 40, I-00044 Frascati, Italy. \\ 2) Università degli Studi di Roma "La Sapienza", Piazzale A. Moro. \\ 3) CERN CH-1211 Genéve 23 F-01631 Switzerland. \\ 4) INFN Sezione di Bari, Via Amendola, 173I-70126 Bari, Italy. \\ 5) Dipartimento Interateneo di Fisica, Via Amendola, 173I-70126 Bari, Italy. \\ 6) Lappeenranta University of Technology, P.O. Box 20 FI-538 1 Lappeenranta, Finland. \\ 7) INFN Sezione di Napoli, Complesso Universitario di Monte Sant'Angelo, edificio 6, 80126 Napoli, Italy. \\ 8) Università di Napoli Federico II, Complesso Universitario di Monte Sant'Angelo, edificio 6, 80126 Napoli, Italy. \\ 9) INFN Sezione di Pavia, Via Bassi 6, 27100 Pavia, Italy and Università degli studi di Pavia, Via Bassi 6, 27100 Pavia, Italy.
}

\begin{abstract}
The status of the CMS RPC Gas Gain Monitoring (GGM) system developed at the Frascati Laboratory of INFN (Istituto Nazionale di Fisica Nucleare) is reported on. The GGM system is a cosmic ray telescope based on small RPC detectors operated with the same gas mixture used by the CMS RPC system. The GGM gain and efficiency are continuously monitored on-line, thus providing a fast and accurate determination of any shift in working point conditions. The construction details and the first result of GGM commissioning are described.
\end{abstract}

PACS: 07.77.Ka; 95.55.Vj; 29.40.Cs

Presented by L. Benussi at the RPC07 - February 2008, Mumbai, India 


\section{Introduction}

Design parameters, construction, prototyping and preliminary commissioning results of the CMS RPC Gas Gain Monitoring (GGM) system are presented [1]. The Resistive Plate Counter (RPC) system is part of the muons detector of the Compact Solenoid Spectrometer (CMS) experiment [2] at the Large Hadron Collider (LHC) collider in CERN (Geneva, Switzerland), with the primary task of providing first level trigger and synchronization. The CMS RPCs are bakelite-based double-gap RPC with strip readout (for construction details see [3] and reference therein) operated with $96.2 \% \mathrm{C}_{2} \mathrm{H}_{2} \mathrm{~F}_{4}-3.5 \%$ Iso- $\mathrm{C}_{4} \mathrm{H}_{10}$ $0.3 \% \mathrm{SF}_{6}$ gas mixture humidified at about $40 \%$. The large volume of the whole CMS RPC system and the cost of gas used make mandatory the operation of RPC in a closedloop gas system (for a complete description see [4]), in which the gas fluxing the gaps is reused after being purified by a set of filters[5].

The operation of the CMS RPC system is strictly correlated to the ratio between the gas mixture components and to the presence of pollution due to contaminants that can be be produced inside the gaps during discharges (i.e. HF produced by $\mathrm{SF}_{6}$ or $\mathrm{C}_{2} \mathrm{H}_{2} \mathrm{~F}_{4}$ molecular break-up and further fluorine recombination), accumulated in the closed-loop or by pollution that can be present in the gas piping system (tubes, valves, filters, bubblers, etc.) and flushed into the gaps by the gas flow. To monitor the presence of these contaminants as well as the gas mixture stability, is therefore mandatory to avoid RPC damage and to ensure their correct functionality.

A monitoring system of the RPC working point due to changes of gas composition and pollution must provide a faster and sensitive response than the CMS RPC system itself in order to avoid irreversible damage of the whole system. Such a Gas Gain Monitoring system monitors efficiency and signal charge continuously by means of a small sized cosmic ray telescope based on RPC detectors. In the following will be briefly described the final setup of the GGM system, its construction details and the first results obtained during its commissioning done at the ISR area (CERN).

\section{The Gas Gain Monitoring System}

The GGM system is composed by the same type of RPC used in the CMS detector but of smaller size $\left(2 \mathrm{~mm}\right.$ Bakelite gaps, $\left.50 \times 50 \mathrm{~cm}^{2}\right)$. Twelve gaps are arranged in a stack located in the CMS gas area (SGX5 building) in the surface, close to CMS assembly hall (LHC-P5). The choice to install the system in the surface instead of underground allows one to profit from maximum cosmic muon rates. In order to ensure a fast response to working point shifts with a precision of $10 \%, 10^{3}$ events are are required, corresponding 
to about 30 minutes exposure time on surface, to be compared with a 100-fold lesser rate underground. The trigger will be provided by four out of twelve gaps of the stack, while the remaining eight gaps will be used to monitor the working point stability.

The eight gaps are arranged in three sub-system: one sub-system (two gaps) will be fluxed with the fresh CMS mixture and its output sent to vent. The second sub-system (three gaps) will be fluxed with CMS gas coming from the closed-loop gas system and extracted before the gas purifiers, while the third sub-system (three gaps) are operated with CMS gas extracted from the closed-loop extracted after the gas filters. The basic idea is to compare the operation of the three sub-system and, if some changes are observed, to send a warning to the experiment. In this way, the gas going to and coming from the CMS RPC detector is monitored by using the two gaps fluxed with the fresh mixture as reference gaps. This setup will ensure that pressure, temperature and humidity changes affecting the gaps behavior do cancel out by comparing the response of the three subsystem operating in the same ambient condition.

The monitoring is performed by measuring the charge distributions of each chamber. The eight gaps will be operated at different high voltages, fixed for each chamber, in order to monitor the total range of operating modes of the gaps. The operation mode of the RPC changes as a function of the voltage applied. A fraction of the eight gaps will work in pure avalanche mode, while the remaining will be operated in avalanche+streamer mode. Comparison of signal charge distributions and the ratio of the avalanche to streamer components of the ADC provides a monitoring of the stability of working point for changes due to gas mixture variations.

\section{Construction and commissioning}

Each chamber of the GGM system consists of a single gap with double sided pad readout: two copper pads are glued on the two opposite external side of the gap. Fig.1 shows a sketch of a chamber whose photos are shown in fig.2; the two foam planes are used to reduce the capacity coupling between the pad and the copper shields. The signal is read-out by a transformer based circuit A3 (Fig.3). The circuit 


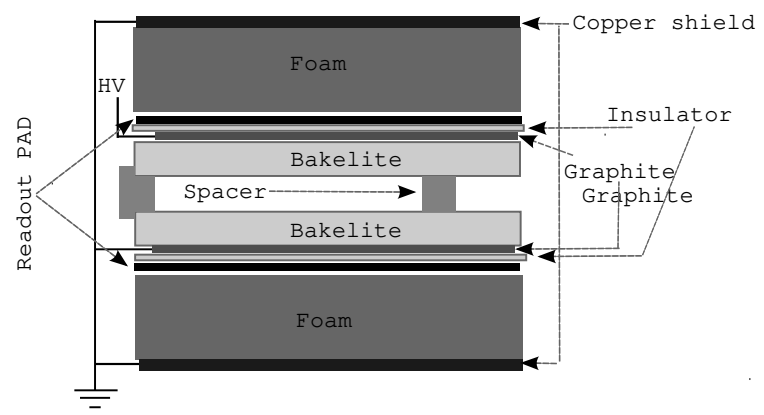

Figure 1: A schematic layout of a GGM chamber

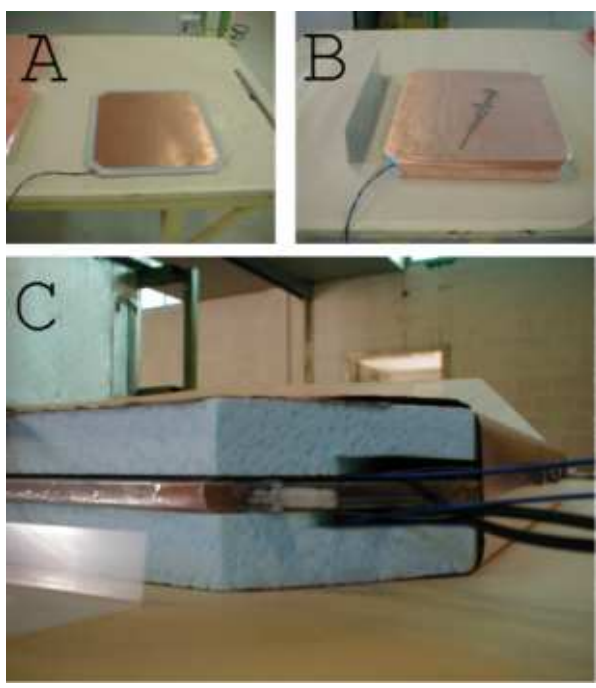

Figure 2: Pictures of a GGM gap and chamber. A) a bare gap with the HV and signal cables. B) a completed chamber. The gap is sandwiched between two foam panels and fully covered with a copper shield. C) a section of a chamber with the two foam panels visible.

allows to algebraically subtract the two signal, which have opposite polarities, and to obtain an output signal with subtraction of the coherent noise, with an improvement by about a factor 4 of the signal to noise ratio. Fig. 4 shows the typical operation mode of the GGM double-pad readout with positive and negative pads pulses, and the output pulse from circuit A3. The output signals from circuit A3 are sent to a CAEN V965 ADC [7] for charge analysis. The GGM has been tested with cosmic rays at LNF and then shipped to CERN for the final commissioning (fig.5 show the final stack assembly). 


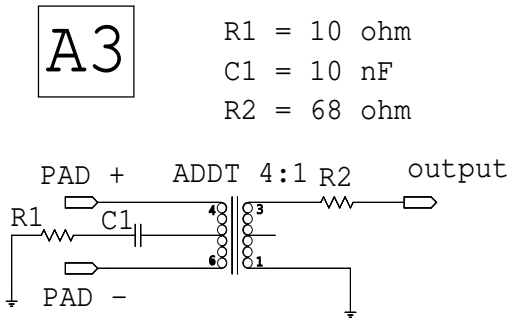

Figure 3: The electric scheme of the read-out circuit providing the algebraic sum of the two pad signal (PAD + and PAD -).

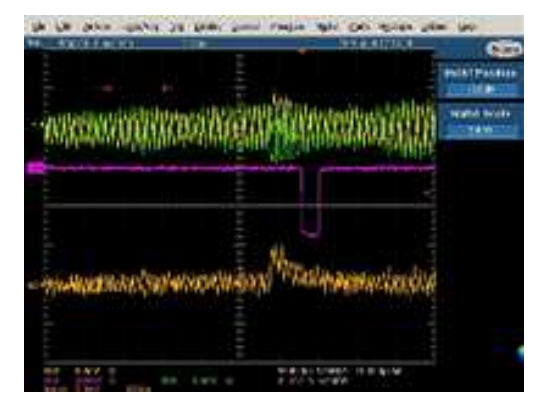

Figure 4: An oscilloscope screen-shot of the two pad signals (upper traces) which are effected by a coherent noise and are barely visible on the screen. In the lower trace the coherent noise is highly reduced by A3 circuit. The vertical scale is the same for both cases $5 \mathrm{mV} / \mathrm{div}$.

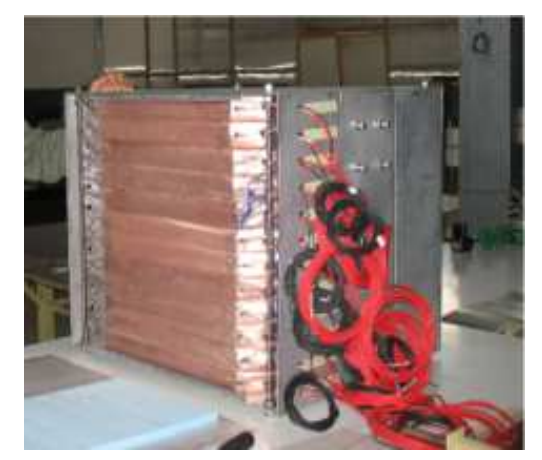

Figure 5: A picture of the GGM system ready to be shipped to CERN. The stack is enclosed into an aluminum box for further shielding.

A typical ADC distribution of a GGM gap is shown in fig.6 for two different effective operating voltage, defined as the high voltage set on the HV power supply corrected for the local atmospheric pressure and temperature. Fig. 6 a) corresponding to $\mathrm{HV}_{e f f}=9.9 \mathrm{kV}$ 
shows a clean avalanche peak well separated from the pedestal. Fig.6 b) shows the charge distribution at $\mathrm{HV}_{\text {eff }}=10.7 \mathrm{kV}$ with two signal regions corresponding to the avalanche and to avalanche+streamer mode.

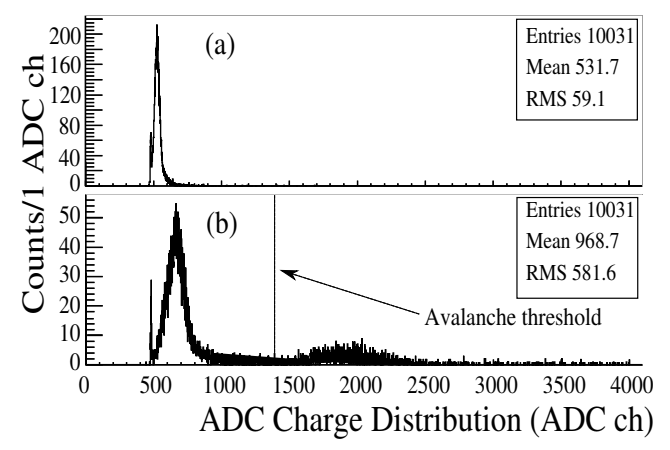

Figure 6: Typical ADC charge distributions of one GGM chamber at two operating voltages. Distribution (a) correspond to $\mathrm{HV}_{\text {eff }}=9.9 \mathrm{kV}$ while distribution (b) to $\mathrm{HV}_{\text {eff }}=10.7 \mathrm{kV}$. In (b) is clearly visible the streamer peak around 1900 ADC channels. The events on the left of the vertical line (1450 ADC channels in this case) are assumed to be pure avalanche events.

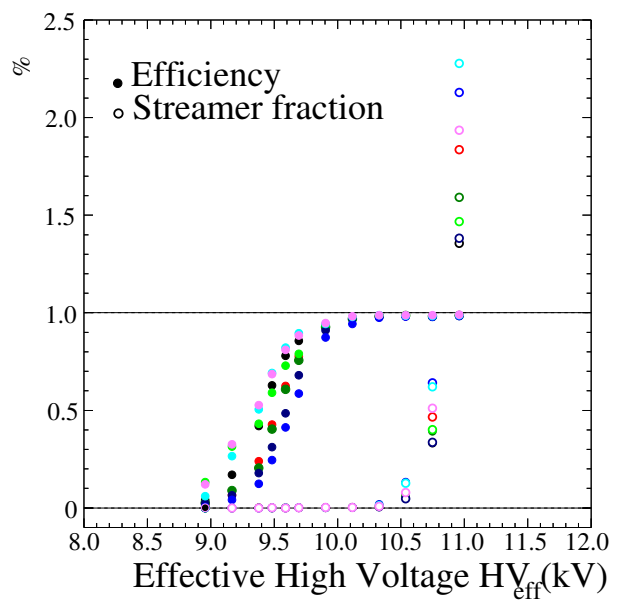

Figure 7: Efficiency plot (full dots) of GGM chambers as a function of $\mathrm{HV}_{\text {eff }}$. The efficiency is defined as the ratio between the number of ADC entries above $3 \sigma_{\text {ped }}$ and the number of acquired triggers. Open dot plots correspond to the streamer fraction of the chamber signal as a function of $\mathrm{HV}_{\text {eff }}$.

Fig.7 shows the GGMS single gap efficiency (full dots), and the ratio between the avalanche and the streamer component (open circles), as a function of the effective high voltage. 
Each point corresponds to a total of 10000 entries in the full ADC spectrum. The efficiency is defined as the ratio between the number of triggers divided by the number of events above $3 \sigma_{\text {ped }}$ over ADC pedestal, where $\sigma_{\text {ped }}$ is the pedestal width. The avalanche to streamer ratio is defined by counting the number of entries in the avalanche (below the ADC threshold (fig. 6 b) and above the pedestal region) and dividing it by the number of streamer events above the avalanche threshold. Both efficiency and avalanche plateau are in good agreement with previous results [6].

In order to determine the sensitivity of GGM gaps to working point shifts, the avalanche to streamer transition was studied by two methods, the charge method and the efficiency method. In the charge method, the mean value of the ADC charge distribution in the whole $\mathrm{ADC}$ range is studied as a function of $\mathrm{HV}_{\text {eff }}$ (fig.8). Each point corresponds to 10000 events in the whole ADC spectrum. In the plot three working point regions are identified

1. inefficiency $\left(\mathrm{HV}_{\text {eff }}<9.7 \mathrm{kV}\right)$;

2. avalanche $\left(9.7 \mathrm{kV}<\mathrm{HV}_{\text {eff }}<10.6 \mathrm{kV}\right.$;

3. avalanche+streamer mode $\left(\mathrm{HV}_{\text {eff }}>10.6 \mathrm{kV}\right)$.

The best sensitivity to working point shifts is achieved in the avalanche+streamer region, estimated to be about $25 \mathrm{ADC} \mathrm{ch} / 10 \mathrm{~V}$ or $1.2 \mathrm{pC} / 10 \mathrm{~V}$.

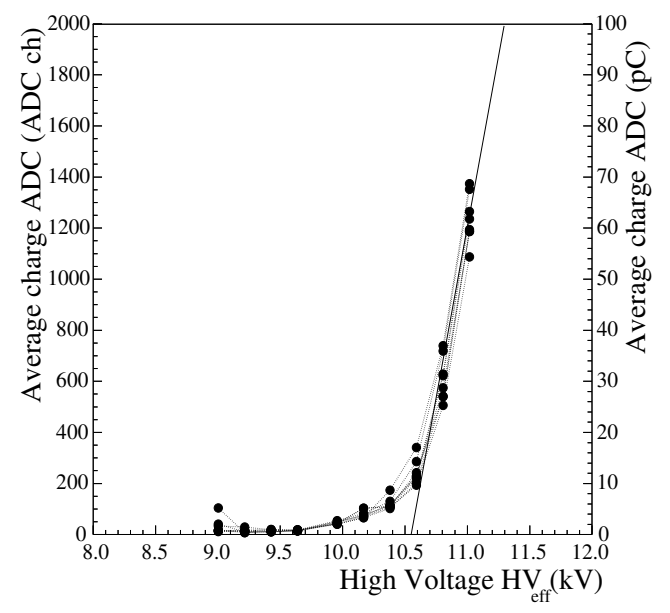

Figure 8: Avarege avalanche charge of the eight monitor chamber signal as a function of $\mathrm{HV}_{\text {eff }}$. The slope is about $25 \mathrm{ADC} \mathrm{ch} / 10$ or $1.2 \mathrm{pC} / 10 \mathrm{~V}$. Each point corresponds to 10000 triggers. 
In the efficiency method, the ADC avalanche event yield is studied as a function of $\mathrm{HV}_{\text {eff }}(9)$. The avalanche signal increases by increasing the $\mathrm{HV}$ applied to the gap, until it reaches a maximum value after which the streamer component starts to increase. The $9.0 \mathrm{kV}-10.0 \mathrm{kV}$ shows a sensitivity to work point changes of approximately $1.3 / \% / 10 \mathrm{~V}$.

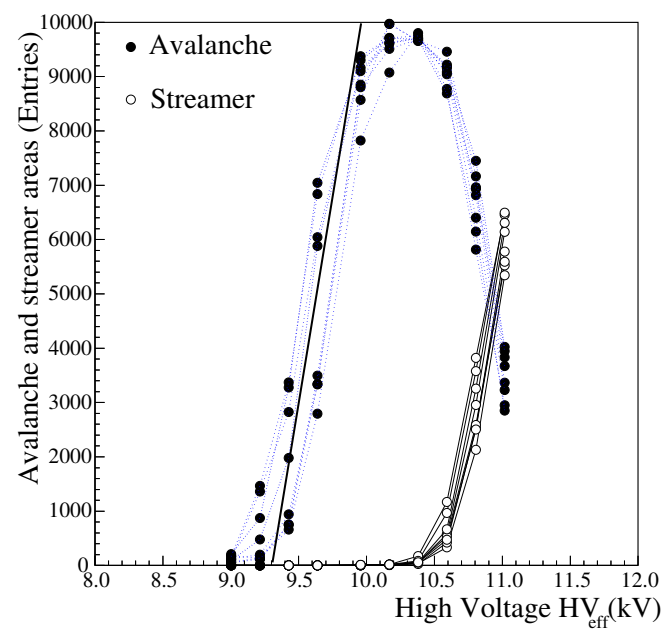

Figure 9: Streamer and avalanche yields as a function of $\mathrm{HV}_{\text {eff }}$. Each point corresponds to 10000 collected triggers. The solid line has a slope of approximately 130 events/10 V corresponding to a sensitivity of $1.3 \% / 10 \mathrm{~V}$.

\section{Conclusions}

The status of the Gas Gain Monitoring System for the CMS RPC Detector has been reported on. The purpose of GGM is to monitor any shift of the working point of the CMS RPC detector. The GGM is being commissioned at CERN and is planned to start operation by the end of 2008. Preliminary results show good sensitivity to working point changes. Further tests are in progress to determine the sensitivity to gas variations.

\section{Acknowledgements}

We warmly thank F. Hahn and the CERN Gas Group for useful discussions and cooperation. 


\section{References}

[1] M. Abbrescia et al., "Gas analysis and monitoring systems for the RPC detector of CMS at LHC", presented by S.Bianco at the IEEE 2006, San Diego (USA), arXiv:physics/0701014.

[2] The CMS Collab., The CMS experiment at the CERN LHC, to appear on Journal of Instrumentation 2008.

[3] A. Colaleo et al., The Compact Muon Solenoid RPC Barrel Detector, these Proceedings.

[4] R. Guida, these Proceedings.

[5] G. Saviano, these Proceedings.

[6] M. Abbrescia et al., Nucl. Instrum. Meth. A 550, 116 (2005).

[7] C.A.E.N. Costruzioni Apparecchiature Elettroniche Nucleari S.p.A. Via Vetraia 11 - 55049 Viareggio (Italy). 